\title{
Expansion of the LOFAR Radboud Air Shower Array
}

\section{K. Mulrey ${ }^{1 *}$ A. Bonardi ${ }^{2}$, S. Buitink ${ }^{1}$, A. Corstanje ${ }^{2}$, H. Falcke ${ }^{2,3,4}$, B. M. Hare ${ }^{5}$, J. R. Hörandel ${ }^{2,3}$, P. Mitra ${ }^{1}$, A. Nelles ${ }^{2,6}$, J. P. Rachen ${ }^{2}$, L. Rossetto ${ }^{2}$, P. Schellart ${ }^{2,7}$, O. Scholten ${ }^{5,8}$, S. ter Veen ${ }^{2,4}$, S. Thoudam ${ }^{2,9}$, T. N. G. Trinh ${ }^{5}$, T. Winchen ${ }^{1}$}

${ }^{1}$ Astrophysical Institute, Vrije Universiteit Brussel, Pleinlaan 2, 1050 Brussels, Belgium,

${ }^{2}$ Department of Astrophysics/IMAPP, Radboud University, P.O. Box 9010, 6500 GL Nijmegen, The Netherlands,

${ }^{3}$ NIKHEF, Science Park Amsterdam, 1098 XG Amsterdam, The Netherlands,

${ }^{4}$ Netherlands Institute of Radio Astronomy (ASTRON), Postbus 2, 7990 AA Dwingeloo, The Netherlands,

${ }^{5}$ KVI-CART, University Groningen, P.O. Box 72, 9700 AB Groningen,

${ }^{6}$ Department of Physics and Astronomy, University of California Irvine, Irvine, CA 92697-4575, USA,

${ }^{7}$ Department of Astrophysical Sciences, Princeton University, Princeton, NJ 08544, USA,

${ }^{8}$ Interuniversity Institute for High-Energy, Vrije Universiteit Brussel, Pleinlaan 2, 1050 Brussels, Belgium,

${ }^{9}$ Department of Physics and Electrical Engineering, Linnéuniversitetet, 35195 Växjö, Sweden

E-mail: kmulreyavub.ac.be

The LOFAR Radboud Air Shower Array (LORA) consists of 20 plastic scintillators and is situated at the core of the LOFAR radio telescope. LORA detects particles from extensive air showers and triggers the read-out of the LOFAR antennas. The dense LOFAR antenna spacing allows for detailed sampling of the radio emission generated in extensive air showers, which yields high precision reconstruction of cosmic ray properties and information about the shower development. We discuss the proposed expansion of LORA, including the addition of scintillator units and the implementation of triggering algorithms that will probe more details of the radio emission and detect lower energy showers without introducing a composition bias, which is important for studying the origin of cosmic rays.

35th International Cosmic Ray Conference - ICRC2017

10-20 July, 2017

Bexco, Busan, Korea

* Speaker. 


\section{Introduction}

As air showers develop in the atmosphere, radio emission is generated through the charge excess effect and the transverse current induced by the magnetic field of the Earth. The resulting pattern of radiation on the ground is highly structured and information about the primary cosmic ray can be extracted from radio data [1]. Radio techniques have been used to reconstruct the energy, geometry, and composition of cosmic rays with high precision [2]. The LOw Frequency ARray (LOFAR) is particularly well suited to study radio emission from cosmic rays because of its dense antenna spacing $[3,4]$. LOFAR is optimal for detecting air showers with energies between $10^{16}$ and $10^{18} \mathrm{eV}$ where the transition from galactic to extra-galactic cosmic rays is expected to exist.

The LOfar Radboud Air shower array (LORA) is deployed in the LOFAR core as a means to trigger the LOFAR array and provide information about the primary cosmic ray based on particle data [5]. LORA data has been used to construct the cosmic ray energy spectrum between $10^{16}$ and $10^{18} \mathrm{eV}$ [6]. Because of the steeply falling cosmic ray spectrum, most triggered events are low in energy, around $10^{16} \mathrm{eV}$, where the radio signal is unlikely to be detectable above background noise. Additionally, a composition bias is introduced in the triggering scheme. We propose to add additional scintillators to the array in the LOFAR core, with the goal of increasing the usability of triggered events.

\section{LORA}

LORA currently consists of 20 plastic scintillators, $0.95 \mathrm{~m} \times 0.95 \mathrm{~m}$ in size, arranged in 5 stations of 4 detectors. The scintillators were previously used in the KASCADE experiment [7]. Each LORA station is associated with a LOFAR low-band antenna (LBA) station and has an interscintillator spacing between $50 \mathrm{~m}$ and $100 \mathrm{~m}$. The entire array spans $300 \mathrm{~m}$ across the LOFAR superterp, as shown in the left panel of Figure 1. LORA provides a trigger for LOFAR antenna readout when a given number of detectors have a strong enough signal to noise ratio. The threshold level for detection roughly corresponds to the amount of energy deposited in a scintillator by one muon. When at least one LORA station detects a signal in at least 3 out of 4 scintillators, information from all the stations is sent to a master computer. A high-level trigger is then formed to read out data from the active LOFAR antennas. Currently, LOFAR is triggered when 13 out of 20 scintillators have a signal. The trigger condition influences the trigger rate and the energy threshold for detection. The current condition ensures that the data read out rate at LOFAR remains manageable while minimizing triggers from events with energy lower than $10^{16} \mathrm{eV}$.

At present, $19 \%$ of triggered events have a detectable cosmic ray radio signal, as determined by the data reduction pipeline [3]. This point is demonstrated in the right panel of Figure 1. The detected flux of cosmic rays with usable radio signals is shown, where the event energies are reconstructed using a fit to a two dimensional radio LDF [2, 8]. Radio signals become detectable above $10^{16.5} \mathrm{eV}$. It is useful to compare events with usable radio signal with all events triggered with LORA. To do this, simulated air showers are used. Details of the simulation process are discussed in Section 3. Simulated flux is scaled to the measured data at $10^{17.5} \mathrm{eV}$ because the time of detection, and therefore flux, is not known, as operating conditions vary and are not continuous. The comparison illustrates that events with energies above $10^{17} \mathrm{eV}$ generally have a strong enough 

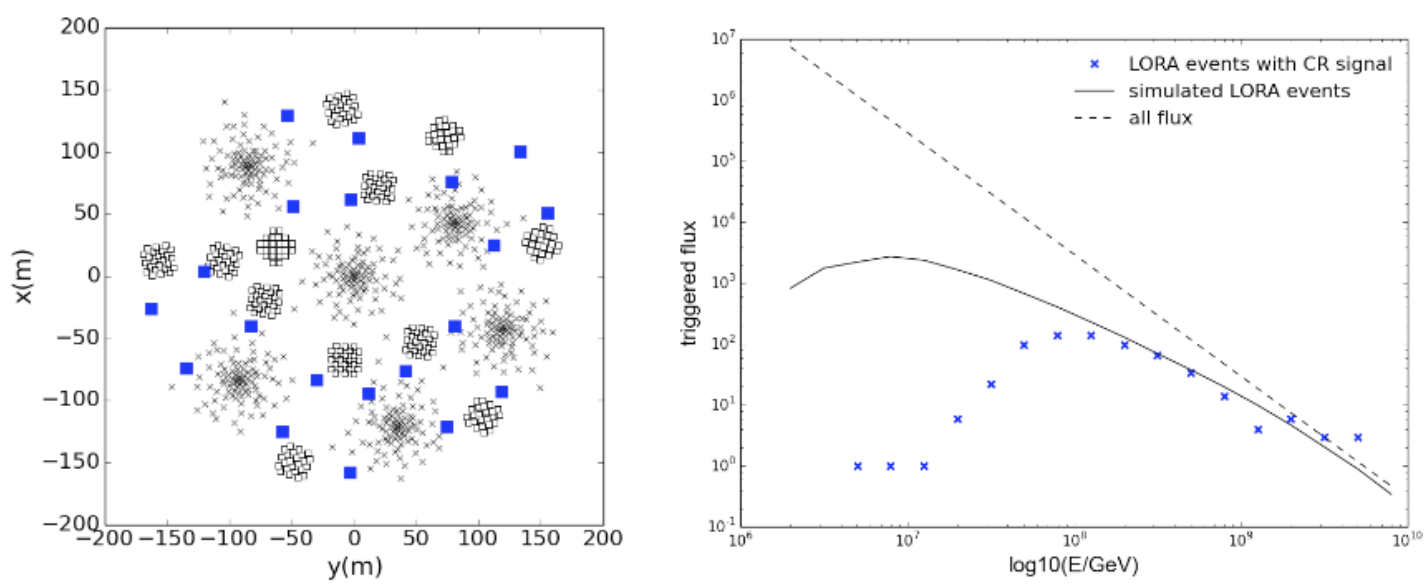

Figure 1: Left: Layout of the LORA array and LOFAR antennas in the superterp. Blue squares represent LORA detectors, black x's represent LBA antennas, and black boxes represent HBA antennas. Right: Measured and simulated cosmic ray fluxes. For measured LORA events, marked with blue crosses, all events with a detectable radio signal are included. For simulated events, the total flux of cosmic rays is multiplied by the probability of triggering LORA and scaled to the measured data at $10^{17.5} \mathrm{eV}$, since the time frame of detection is unknown. $19 \%$ of triggered events are above $10^{16.5} \mathrm{eV}$, and therefore have a likely detectable radio signal. The dashed line represents the total cosmic ray flux.

radio signal to be detected, and that the majority of triggered events do not have a detectable radio signal.

In order to trigger on only the high energy showers, the number of detectors necessary to form a LOFAR trigger could be increased. However, this reduces the trigger rate to an unnecessarily low level. Additionally, there is a bias introduced by the number of detectors required for a trigger. Since light primaries generally interact later in the atmosphere than heavy primaries, more shower particles reach the ground detection level. Therefore, light primary cosmic rays are more likely to trigger LORA than heavy primaries, as shown in Figure 2. Here, the probability of an event within $250 \mathrm{~m}$ of the LOFAR core triggering is plotted as a function of the number of detectors required for a LOFAR trigger. Below $10^{17} \mathrm{eV}$, the probability of triggering 13 detectors is different for proton and iron primaries, and so a bias is introduced. It would be optimal to have a clean sample for energies down to $10^{16.5} \mathrm{eV}$, which is interesting for source transition studies. In practice, some detected events have cores outside of $250 \mathrm{~m}$ radius, and so composition bias has to be studied on an event by event basis.

\section{Simulations and Design consideration}

Simulations have been used to determine the optimal layout of the LORA expansion. Air showers are generated with CORSIKA [9], using QGSJET II-04 [10] for energies above $200 \mathrm{GeV}$ and FLUKA [11] for lower energies. Showers for proton and iron primaries are simulated for energies between $10^{15}$ and $10^{19} \mathrm{eV}$ and for zenith angles between $0^{\circ}$ and $45^{\circ}$. The flux is weighted to a -3 spectral index. Thinning is used at a level of $10^{-6}$ [12]. For each shower, all ground level particles are projected into the shower plane, and collected in radial bands of $5 \mathrm{~m}$. A GEANT4 [13] 

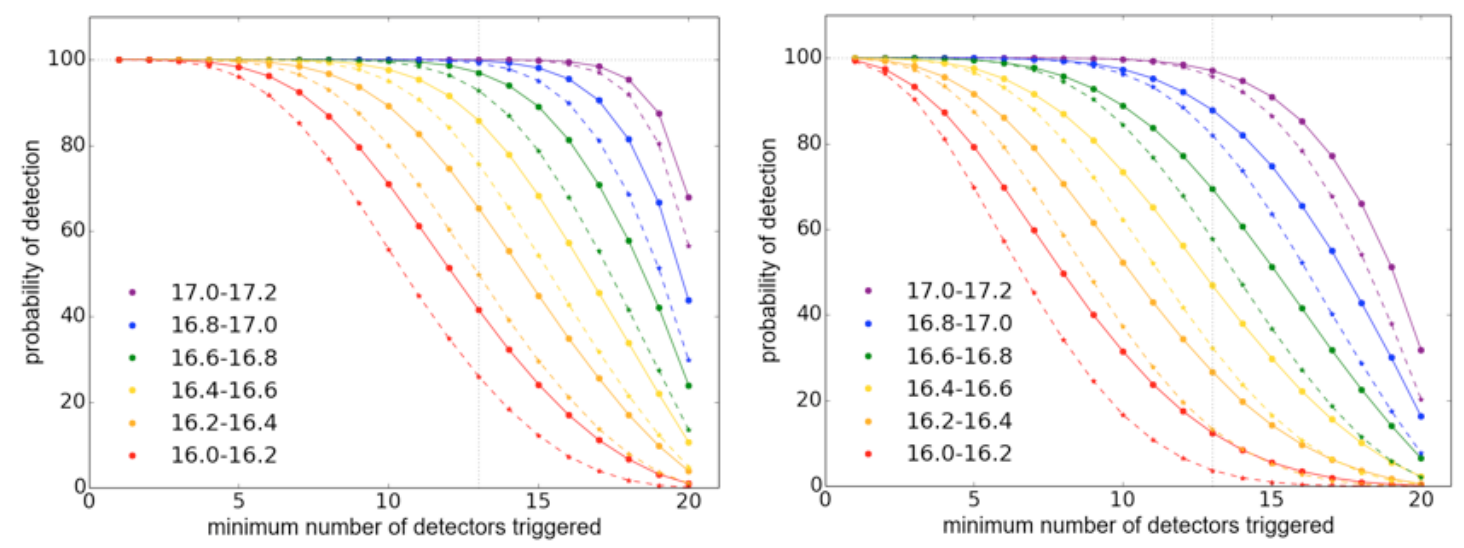

Figure 2: Probability of triggering a minimum number of detectors. Solid lines represent proton showers and dashed represent iron showers. Left: Zenith angles between $0^{\circ}$ and $30^{\circ}$. Right: Zenith angles between $30^{\circ}$ and $45^{\circ}$. The different colors represent different energy bands, as indicated in the legend as $\log ($ energy/eV).

simulation with realistic LORA scintillator geometry is used to convert the particles at ground level into an energy deposit per $\mathrm{m}^{2}$ as a function of radius to the shower core in the shower plane. Each shower is then given 100 random positions within $2000 \mathrm{~m}$ of the center of the superterp. This distance is chosen to ensure that not all showers trigger LORA. The positions of the detectors are projected into the shower plane and assigned an energy deposit based on their position relative to the shower core.

For each shower, the number of detectors with signal is calculated. The current LORA signal threshold is the average energy deposited by one muon. Therefore, the simulated detectors are considered to contain a detectable signal if they contain the energy deposit of at least one muon, which from simulation is $6.4 \mathrm{MeV}$. The total energy in the detector is divided by this number, and then the final muon content of the detector is drawn from a Poisson distribution.

The placement of additional detectors on the ground is dictated by logistical considerations. Detectors can only be placed on land around current LOFAR stations, and must not interfere with current operations. Digitizing electronics are most convenient to use in sets of 2 or 4 . For this reason, additional stations consist of 4 scintillators and are simulated in the locations of LOFAR LBA fields in rings of radius $50 \mathrm{~m}$, as shown in Figure 3. In total 20 detectors are available for the expansion.

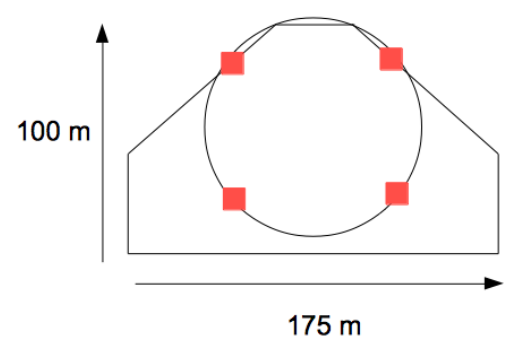

Figure 3: Simulated positions of detectors in additional stations. 
New LORA layouts are achieved by adding new stations of four scintillators. This document includes 6 arrangements of note, as seen in Figure 4. The naming system indicates the total number of scintillators included. These arrangements were chosen to study the effects of detector spacing and the total number of scintillators used. For example, arrangement $40 \mathrm{~b}$ has 5 new stations with farther spacing than $40 \mathrm{a}$.
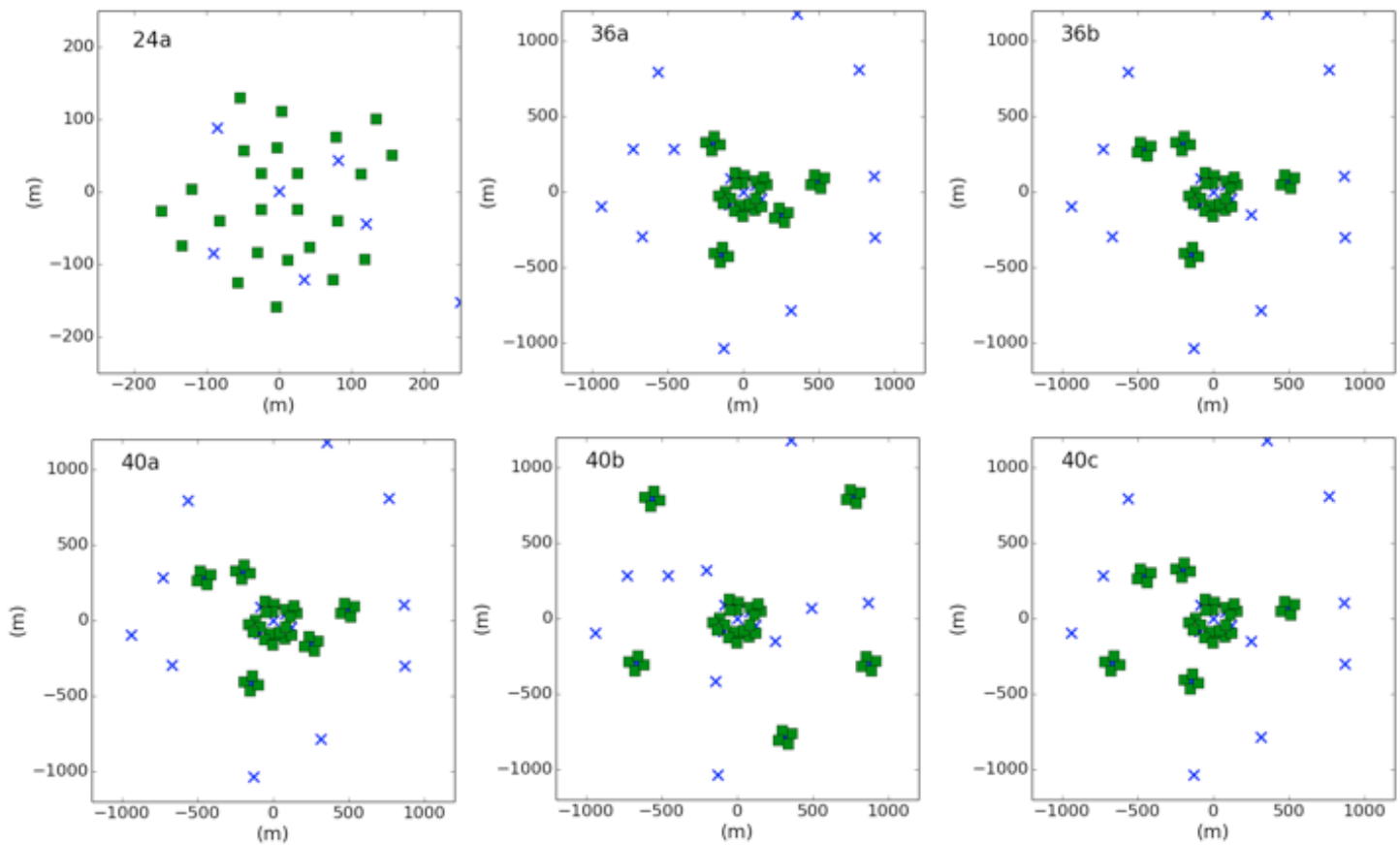

Figure 4: Arrangements of simulated LORA arrangements. Green squares represent scintillators, and blue crosses mark the center of LOFAR LBA stations. Notice the scale on arrangement 24a is different to show the infill of the current LORA arrangement.

For 19 energy bins between $10^{15} \mathrm{eV}$ and $10^{19} \mathrm{eV}$, the probability of a minimum number of detectors having a signal is determined. This probability is multiplied by the cosmic ray flux in each energy bin. From this information, we study the trigger rate, triggered flux, and probability of triggering a minimum number of detectors. The aim is to increase the number of unbiased events above $10^{16.5} \mathrm{eV}$, which are more likely to have a detectable radio signal, while maintaining the current event rate.

\section{Results}

We first look at the expected trigger rate for each arrangement. The left panel of Figure 5 shows the trigger rate in $\mathrm{Hz}$ for a minimum number of detectors triggered. For example, the current trigger condition is 13 detectors with a signal, an the current trigger rate is $\sim 0.8$ events/hour. LORA stations outside the superterp have 4 scintillators each, so when the trigger condition is 4 or fewer, the trigger rate for arrangements with more stations increases, while 24a remains similar to the current LORA rate. As the trigger condition increases, 24a has a high trigger rate than 
the others, since one air shower that triggers at least 8 detectors will likely also include the infill. The conclusion is that the trigger rate for any LORA extension remains manageable, although the trigger condition may need to be increased.

Secondly, we look at the number of cosmic rays that will be detectable in different energy bins. The right panel of Figure 5 shows the triggered flux multiplied by energy for different arrangements. Since the flux depends on the trigger conditions, here we have used the condition that keeps the trigger rate at 1 event/hour. For condition 24a, 36a, and 40b it is 15 detectors. For 36b, and $40 \mathrm{c}$ this is 13 detectors, and for the current set up and 40a it is 12 detectors. Arrangement 24a collects a larger flux at low energies and offers no more improvements at high energies. Arrangement $40 \mathrm{~b}$, which is the option featuring the most widely spread out stations, performs similarly to LORA at low energies and then shows an improvement at the highest energies, where the particle footprint is large enough to encompass all the detectors. Arrangements 40c, 40a, 36a, and 36b show improvement in the whole range from $10^{16}$ to $10^{18} \mathrm{eV}$, with $40 \mathrm{c}$ performing the best.
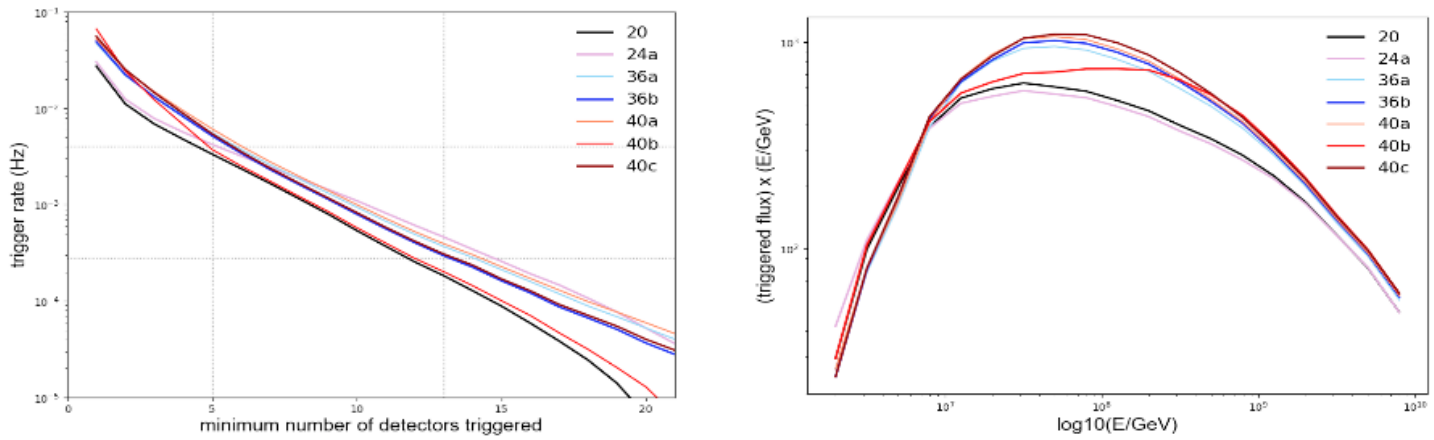

Figure 5: Left: Event rate for a minimum number of detectors required for a trigger. Right: CR flux multiplied by the probability of triggering. The trigger condition is chosen to keep the rate under 1 event per hour, and so different detector arrangements have different conditions.

Finally, we look at the probability of detection for different trigger conditions. For an area of $250 \mathrm{~m}$ around the center of the superterp, where $95 \%$ of event cores are currently located, the probability of detecting proton and iron showers between $10^{16.4}$ and $10^{16.6} \mathrm{eV}$ is shown in Figure 6. This is the lowest energy band of interest, and higher energies will already have less intrinsic bias. Only arrangements $24 \mathrm{a}, 36 \mathrm{~b}$, an 40c are shown. At a trigger condition of 13 out of 20 detectors, an increase in probability of detection is already evident, particularly for zenith angles below $30^{\circ}$. Again, since not all cores are contained in the $250 \mathrm{~m}$ radius, it is necessary to determine bias on a case by case basis, however, increasing the probability of detection for both heavy and light primaries lessens the chance of a biased detection.

\section{Science Outlook}

Considering the cases of arrangements 24a, 36b, and 40c, we can determine the increase in events above $10^{16.5} \mathrm{eV}$. Table 1 contains the percentage of simulated, triggered events above $10^{16.5} \mathrm{eV}$ based on the trigger condition that yields one event per hour, and the percent increase over the current LORA performance. The $24 \mathrm{a}$ detector arrangement performs worse than the cur- 

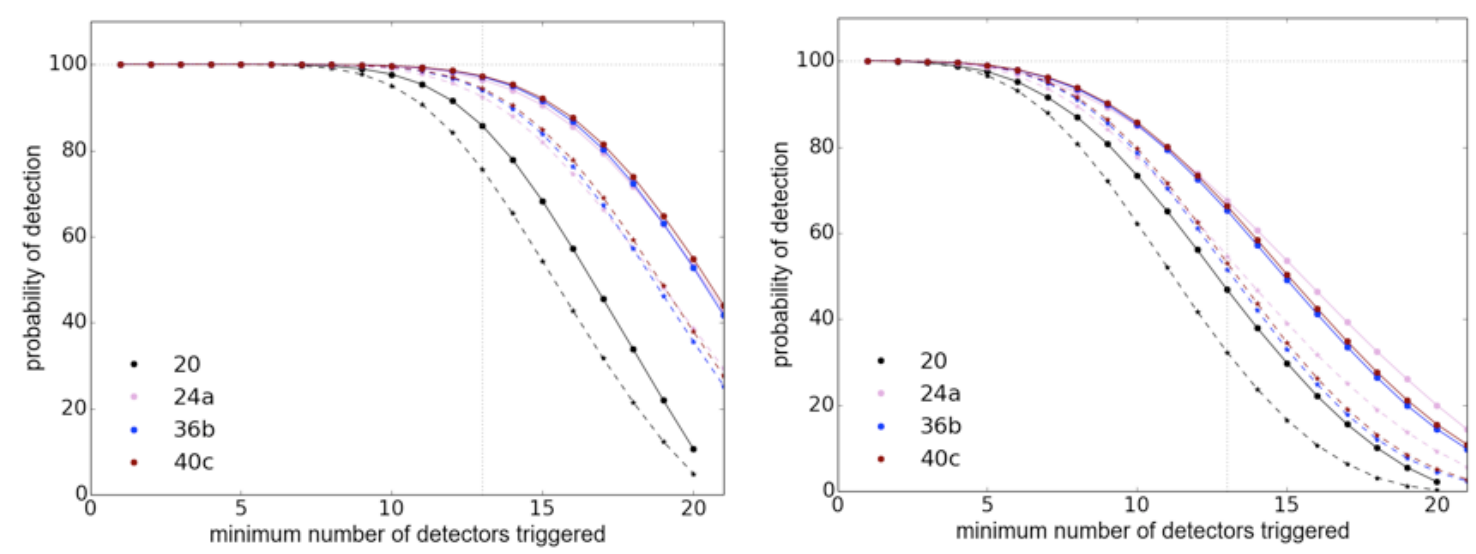

Figure 6: Probability of triggering a minimum number of detectors. Solid lines represent proton and dashed represent iron. Left: Zenith angles between $0^{\circ}$ and $30^{\circ}$. Right: Zenith angles between $30^{\circ}$ and $45^{\circ}$. The different colors represent different detector arrangements, all in the energy bin $10^{16.4}-10^{16.5}$.

rent set up, as lower energy showers show a signal in more detectors. The $36 \mathrm{~b}$ and $40 \mathrm{c}$ arrangements both have a significant increase in good events.

\begin{tabular}{||ccc||}
\hline Det. Arr. & \% good events & \% increase \\
\hline \hline 20 & 19.0 & 0 \\
$24 \mathrm{a}$ & 17.3 & -10 \\
$36 \mathrm{~b}$ & 26.7 & 40.5 \\
$40 \mathrm{c}$ & 27.6 & 45.3 \\
\hline
\end{tabular}

Table 1: Percentage of triggered events above $10^{16.5} \mathrm{eV}$, and the percent increase over current performance.

In addition to the increase in good events, there is other scientific value in expanding LORA. Showers with cores outside the superterp will trigger LOFAR, which allows the dense antenna area in the superterp to sample the fringes of the radio footprint. Since the radio emission outside the Cherenkov cone is less compressed there, the radio pulses contain information about the early development of the shower. Additionally, more complicated trigger algorithms are possible. For example, requiring a specific subset of detectors to contain a signal, rather than a simple minimum number of detectors, allows for the possibility of triggering on specific shower geometries with desirable qualities for radio emission, such as larger zenith angles where the radio footprint is broader. In summery, the expansion of LORA increases the amount of data with a quality radio signal, and offers further possibilities of probing the radio footprint.

\section{Acknowledgements}

The LOFAR cosmic ray key science project acknowledges funding from an Advanced Grant of the European Research Council (FP/2007-2013) / ERC Grant Agreement n. 227610. The project has also received funding from the European Research Council (ERC) under the European UnionâĂŹs Horizon 2020 research and innovation programme (grant agreement No 640130). 
We furthermore acknowledge financial support from FOM, (FOM-project 12PR3041-3) and NWO (Top Grant 614-001-454, and Spinoza Prize SPI 78-409). AN is supported by the DFG (research fellowship NE 2031/1-1).

LOFAR, the Low Frequency Array designed and constructed by ASTRON, has facilities in several countries, that are owned by various parties (each with their own funding sources), and that are collectively operated by the International LOFAR Telescope foundation under a joint scientific policy.

\section{References}

[1] Tim Huege. Radio detection of cosmic ray air showers in the digital era. Physics Reports, 620:1-52, 2016.

[2] S. Buitink et al. Method for high precision reconstruction of air shower $\mathrm{X}_{\max }$ using two-dimensional radio intensity profiles. Phys. Rev. D, 90(8), 2014.

[3] P. Schellart et al. Detecting cosmic rays with the LOFAR radio telescope. Astronomy and Astrophysics, 560(A98), 2013.

[4] M. P. van Haarlem et al. LOFAR: The LOw-Frequency ARray. Astronomy and Astrophysics, 556:56, 2013.

[5] S. Thoudam et al. LORA: A scintillator array for LOFAR to measure extensive air showers. Nucl.Instrum.Meth, A767:339-346, 2014.

[6] S. Thoudam et al. Measurement of the cosmic-ray energy spectrum above $10^{16} \mathrm{eV}$ with the LOFAR Radboud Air Shower Array. Astroparticle Physics, 73:34-43, 2016.

[7] T. Antoni et al. (KASCADE Collaboration). The cosmic-ray experiment KASCADE. Nuclear Instruments and Methods A, 513:490, 2003.

[8] A. Nelles et al. A parameterization for the radio emission of air showers as predicted by CoREAS simulations and applied to LOFAR measurements. Astropart.Phys., 60:13-24, 2015.

[9] D. Heck et al. CORSIKA: A Monte Carlo code to simulate extensive air showers. Report FZKA, 6019, 1998

[10] S. Ostapchenko. Monte Carlo treatment of hadronic interactions in enhanced Pomeron scheme: QGSJET-II model. Phys. Rev. D, 83(014018), 2011.

[11] A. Ferrari et al. FLUKA: a multi-particle transport code. CERN-2005-10, INFN/TC_05/11, 2005.

[12] M. Kobal et al. (Pierre Auger Collaboration). A thinning method using weight limitation for air-shower simulations. Astropart.Phys., 15:259-273, 2001.

[13] S. Agostinelli et al. GEANT4 -a simulation toolkit. NIMPA, 506(3):250-303, 2003. 